\title{
PEGADA DE CARBONO ASSOCIADA AO PROCESSO DE PASTEURIZAÇÃO DE SORVETES
}

Thiago Freire Melquíades*

Monica Carvalho*

Yuri Rommel Vieira Araújo"***

Luiz Moreira Coelho Junior ${ }^{* * * * *}$

RESUMO: Houve uma elevada demanda mundial por combustíveis fósseis para a geração de energia a partir da Revolução Industrial e isso contribuiu para o aumento nos níveis de emissão de poluentes ao longo dos anos. O aproveitamento da energia solar para processos de calor na indústria surgiu como uma alternativa para substituição do uso dos combustíveis fosseis, minimizando os níveis de emissão de gases de efeito estufa. Este estudo aplicou a metodologia da Avaliação de Ciclo de Vida para quantificar a pegada de carbono associada ao processo de pasteurização em uma empresa de fabricação de sorvetes e outros gelados comestíveis em João Pessoa (Paraíba), comparando a substituição do queimador atmosférico de gás liquefeito de petróleo por um sistema de aquecimento solar. Utilizou-se o software SimaPro ${ }^{\circledR}$ para o desenvolvimento do estudo, com método de avaliação de impacto ambiental IPCC 2013 GWP 100a. Os resultados indicaram que o sistema de aquecimento solar proporcionou menor pegada de carbono em comparação ao queimador atmosférico de gás liquefeito de petróleo, e que possui potencial para mitigação de mudanças climáticas.

PALAVRAS-CHAVE: Avaliação de ciclo de vida; Energia solar; Indústria alimentícia; Sistema de aquecimento solar.

\section{CARBON FOOTPRINT ASSOCIATED WITH ICE CREAM PASTEURIZATION PROCESS}

ABSTRACT: In the wake of the Industrial Revolution world demand of fossil fuel for the generation of energy has always been high and contributed towards an

\footnotetext{
Programa de Pós-Graduação em Energias Renováveis (PPGER). Universidade Federal da Paraíba (UFPB) João Pessoa. Administrador, Mestre em Energias Renováveis, Brasil.

** Eng. Eletricista, Dr ${ }^{\mathrm{a}}$, Docente do Departamento de Engenharia de Energias Renováveis Universidade Federal da Paraíba (UFPB), João Pessoa, Brasil. E-mail: monica@cear.ufpb.br

**** Eng. Florestal, Mestre em Energias Renováveis Universidade Federal da Paraíba (UFPB), João Pessoa, Brasil.

***** Economista, Dr., Docente do Departamento de Engenharia de Energias Renováveis (DEER), Brasil.
} 
increase in the levels of pollutant emission over the years. The use of solar energy for industrial heating processes has been an alternative to substitute fossil fuel and minimize the levels of greenhouse gas emissions. The Evaluation of the Life Cycle methodology has been employed to quantify carbon footprint associated with the pasteurization process in an ice cream factor and other ice derivates in João Pessoa PB Brazil. The study also compares the replacement of petroleum liquefied gas air burner by solar heating system. SimaPro® was employed for the development of current analysis through IPCC environmental 2013 GWP 100a impact evaluation. Results indicate that solar heating system provided the lowest carbon footprint rate when compared to gas burner. It has also the capacity to lessen climate changes.

KEY WORDS: Evaluation of the life cycle; Solar energy; Food industry; Solar heating system.

\section{INTRODUÇÃO}

Houve uma elevada demanda mundial por combustíveis fósseis para a geração de energia a partir da Revolução Industrial, o que contribuiu para o aumento nos níveis de emissão de poluentes ao longo dos anos (International Energy Agency - IEA, 2014a). Em 2012, a matriz mundial de fontes de energia primária alcançou $81,7 \%$ de combustíveis fósseis, que incluem o petróleo com 31,4\%, carvão mineral com 29\% e o gás natural com 21,3\% (IEA, 2014b).

Em 2014, o Brasil obteve 55,5\% de toda a produção de energia primária oriunda de combustíveis fósseis, como o petróleo (42,8\%), o gás natural (11,6\%) e o carvão mineral (1,1\%) (EPE, 2015). Considerando o período de 2013 a 2014, o Brasil alcançou um incremento de $11,4 \%$ na produção de petróleo, 13,2\% na produção de gás natural e uma redução de 7,7\% na produção de carvão mineral (EPE, 2015).

De acordo com a Associação Brasileira de Refrigeração, Ar Condicionado, Ventilação e Aquecimento - ABRAVA (2015), uma alternativa para o uso de combustíveis fósseis é o aproveitamento da energia solar para redução de custos e impactos ambientais em empresas. Em 2013, apenas 3\% das vendas de Sistemas de Aquecimento Solar (SAS) foram destinadas a aplicações industriais no Brasil (ABRAVA, 2015). Em 2014, essa porcentagem subiu para 17\%, sendo a maior participação das vendas no segmento residencial (51\%), seguido dos programas habitacionais (16\%) 
e do comércio e serviço (16\%) (ABRAVA, 2015).

A Agência Internacional de Energia (IEA) (2013) afirmou que o uso de SAS representa maior economia em termos equivalentes de petróleo e dióxido de carbono $\left(\mathrm{CO}_{2}\right)$. Corroborando esta afirmação, Schnitzer, Brunner e Gwehenberger (2006) mostram que o aproveitamento da energia solar para processos de calor na indústria substitui o uso de fontes de energia fósseis, minimizando os níveis de emissão de $\mathrm{CO}_{2}$, que é um dos compostos químicos que mais contribuem para o efeito estufa.

A Avaliação de Ciclo de Vida (ACV) é utilizada para avaliar os potenciais impactos ambientais associados a um processo, produto, bem ou serviço e consiste em uma metodologia já consolidada e validada. É uma ferramenta analítica de gestão ambiental que analisa desde a extração da matéria-prima, incluindo fabricação, transporte, utilização, manutenção e o seu destino final (ABNT, 2014a; ABNT, 2014b). A ACV pode avaliar sistematicamente vários impactos ambientais associados a uma atividade, ao longo de seu ciclo de vida, incluindo saúde humana, uso de recursos naturais e consequências aos ecossistemas (GUINÉE, 2001; GUINÉE, 2002). A pegada de carbono pode ser considerada uma aplicação especial da ACV (subconjunto) que foca especificamente nas emissões de gases de efeito estufa (GEE), e a principal vantagem da pegada de carbono em comparação a uma ACV completa é a facilidade de sua comunicação. O público já está mais familiarizado com o termo de emissões de GEE, e assim a pegada de carbono consegue criar uma conexão com uma das prioridades ambientais reconhecidas (aquecimento global) (ARAÚJO et al., 2018; GRILO et al., 2018; NEVES et al., 2018). Este tipo de análise ambiental consegue identificar os impactos referentes à produção e uso de um produto, mostrando alternativas para um melhor desempenho ambiental, e influenciando na tomada de decisões para escolha de produtos e processos (MACHADO, 2011; CARVALHO; FREIRE; MAGNO, 2015; FREIRE et al., 2015; MEDEIROS; CARVALHO; FREIRE, 2015; CARVALHO; ABRAHÃO, 2017).

A gestão sustentável pode ser aplicável às empresas de fabricação de sorvete e outros gelados comestíveis, por apresentar relevância para a indústria de transformação no Brasil. Em 2013, essas empresas representaram 6,31\% do setor alimentício, considerado a quarta maior atividade industrial (BRASIL, 2015). Em 
2014, a produção foi de 1.188 bilhões de litros de sorvetes, com um crescimento de 10,6\% em relação ao ano anterior e o consumo per capita passou de 5,4 litros por habitante, em 2013, para 5,9 em 2014, representando um crescimento anual de 9,3\% (Indústria Alimentar de Congelados, Supercongelados, Sorvetes, Concentrados e Liofiliados no Estado de São Paulo - SICONGEL, 2015). O faturamento nacional das empresas de sorvetes passou de $\mathrm{R} \$ 9.813$ bilhões em 2013 , para $\mathrm{R} \$ 11.820$ bilhões em 2014, um incremento anual de 20,5\% (SICONGEL, 2015).

$\mathrm{Na}$ Paraíba, houve um aumento de 15,4\% no número de empresas de fabricação de sorvete e outros gelados comestíveis ativas, passando de 33 empresas, em 2012, para 39, em 2013 e ocupando a terceira posição no setor alimentício do Estado com participação de 4,7\% (BRASIL, 2015). Ainda segundo o Ministério do Trabalho e Emprego (BRASIL, 2015), entre 2012 e 2013, o número de empregados cresceu $16,8 \%$ nas empresas de fabricação de sorvete e outros gelados comestíveis, passando de 306 para 368 funcionários.

Entre os processos industriais utilizados pelas empresas de fabricação de sorvete e outros gelados comestíveis, está o de pasteurização. De acordo com a resolução RDC 267 de 25 de setembro de 2003 (BRASIL, 2003), os produtos gelados comestíveis à base de leite e/ou ovos devem passar pelo processo de pasteurização por meio de tratamento térmico (aquecimento e resfriamento) por tempo determinado. Esse processo geralmente utiliza combustíveis fósseis como o Gás Liquefeito de Petróleo - GLP e o gás natural, que contribuem para o aumento dos níveis de emissão de gases de efeito estufa.

Desta forma, fazendo uso da metodologia da Avaliação de Ciclo de Vida, este artigo quantificou a pegada de carbono associada ao processo de pasteurização em uma empresa de fabricação de sorvetes e outros gelados comestíveis em João Pessoa, comparando a substituição do queimador atmosférico de GLP por um Sistema de Aquecimento Solar. 


\section{MATERIAIS E MÉTODOS}

\subsection{CARACTERIZAÇÃO DO ESTUDO DE CASO}

Este é um estudo de caso de uma empresa de fabricação de sorvetes e outros gelados comestíveis localizada em João Pessoa, Paraíba, Brasil. A empresa foi criada no ano 2000, e em 2015 possuía 20 funcionários (8 na produção, 2 no administrativo e 10 divididos entre auxiliares, vendedores e cozinheiros), considerada uma microempresa. Os produtos comercializados pela empresa são picolé, açaí e sorvete.

As informações relativas aos recursos, materiais e energia consumidos foram obtidas por meio de visitas técnicas à empresa e entrevistas com os diretores, por meio de um questionário semi-estruturado com questões quali-quantitativa, visando observar o modelo organizacional e as etapas de produção, realizado no mês de junho de 2015.

Para a elaboração e o levantamento das informações referentes ao processo produtivo do sorvete, foi tomado como base o processo de fabricação indicado pelo Serviço Brasileiro de Apoio às Micro e Pequenas Empresas (SEBRAE, 2012), em que os principais equipamentos para a instalação de uma fábrica de sorvetes e picolé são: despolpadeira, dosadora, homogeneizador, pasteurizador e câmara frigorífica.

\subsection{AVALIAÇÃO DE CICLO DE VIDA}

A Avaliação de Ciclo de Vida (ACV) descreve de forma criteriosa todos os consumos e rejeitos do objeto de estudo, proporcionando maior confiabilidade na avaliação de impactos ambientais. A metodologia de uma ACV é regida por normas internacionais da Organização Internacional de Normalização (International Organization for Standardization, ISO), que tratam dos princípios gerais e diretrizes, por meio de um inventário de entrada e saída de materiais e energia em um sistema produtivo (ISO 14040, 2006; ISO 14044, 2006).

No Brasil, a ABNT possui as versões NBR ISO 14040 e 14044 (ABNT NBR ISO 14040, 2014a; ABNT NBR ISO 14044, 2014b), que expõem que os benefícios da ACV podem fornecer elementos importantes para a tomada de decisões em uma empresa. O processo de ACV segue os princípios estabelecidos nas normas (Figura $1)$. 


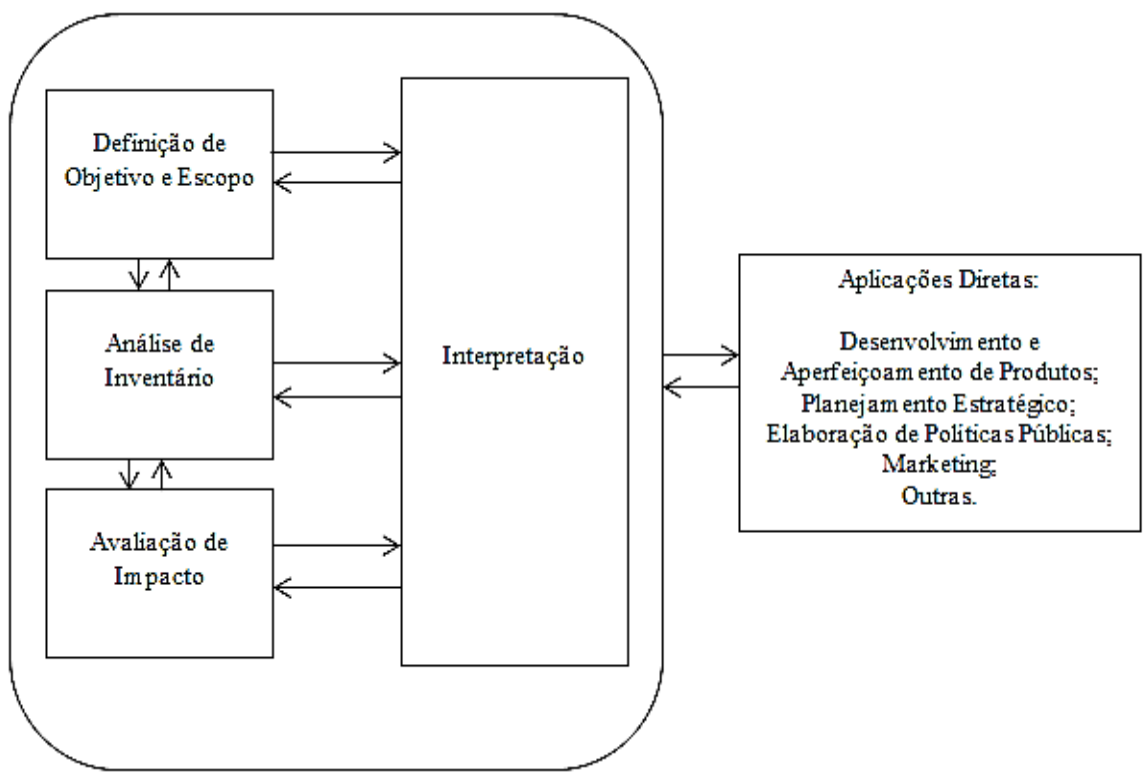

Figura 1. Esquema da Avaliação do Ciclo de Vida. Fonte: ABNT NBR ISO 14040 (2014a).

A descrição da ACV segue a ABNT (2014a; 2014b): inicia-se com a definição do objetivo e do escopo, que consistem nos fatores motivadores e pretendidos do estudo, além do público-alvo a ser atendido e a escolha do produto a ser estudado, juntamente com outros elementos como pressupostos, limitações, unidades do sistema, fronteiras etc. $\mathrm{O}$ procedimento de análise de inventário representa $\mathrm{o}$ conjunto de informações formadas por dados de entradas e saídas do processo em estudo, por exemplo: consumo energético, matéria-prima, resíduos, emissões para o solo, ar, água etc.

A avaliação dos impactos busca entender e associar cada elemento do processo estudado com os indicadores e modelos de caracterização, ou seja, trata-se da classificação dos resultados e sua respectiva definição. A interpretação é formada pela conclusão da análise do inventário, exposição das limitações enfrentadas e possíveis recomendações. Esse elemento proporcionará insumos para a tomada de decisões que irão resultar no desenvolvimento de aplicações diretas, como o aprimoramento de produtos, as estratégias empresariais, o desenvolvimento de 
políticas públicas, o marketing, entre outras.

A aplicação da ACV permite diversas interpretações acerca de um processo ou produto, por exemplo. A amplitude da metodologia está nas averiguações de cada elemento considerado, verificando os potenciais impactos, além de permitir modificações entre esses elementos, observando a sensibilidade nos resultados.

\subsection{DEFINIÇÃO DO ÂMBITO DE ANÁLISE}

O processo analisado é a pasteurização: o fluido é aquecido a uma temperatura de $80{ }^{\circ} \mathrm{C}$ e depois é resfriado a temperatura de $4{ }^{\circ} \mathrm{C}$ ou inferior. Esse procedimento é necessário para o tratamento térmico de derivados do leite e ovos (BRASIL, 2003). O processo de aquecimento alvo deste artigo é realizado por meio de um queimador atmosférico de passagem (Boiler) que apresenta um consumo de $6.41 \mathrm{~kg} / \mathrm{h}$ de GLP.

O volume útil de água para funcionamento do queimador atmosférico é de 185 litros e uma vez que é atingido, as perdas anuais de água são mínimas por se tratar de um sistema fechado. $O$ processo de pasteurização apresenta uma utilização média de 34 horas mensais.

Para comparação com o sistema de referência (Boiler a GLP), selecionouse um SAS. De acordo com a NBR 15569 (ABNT, 2008), um SAS é constituído por coletores solares que convertem a energia radiante em térmica, e em um reservatório térmico que acumula a água quente. O sistema pode apresentar circulação de água natural ou termossifão (alteração na densidade do fluido de trabalho do fluido) ou forçado por meio de moto-bombas (ABNT, 2008).

Para o estudo ambiental, foi considerada a utilização de cada sistema durante um ano para comparação das pegadas de carbono. O ciclo de vida ao qual a unidade funcional está ligada inclui a fabricação dos sistemas e o uso dos recursos necessários para a operacionalização do processo de pasteurização, por ano. 


\subsection{INVENTÁRIO DOS PROCESSOS ENVOLVIDOS}

Os processos utilizados no inventário incluem desde a extração da matériaprima, manufatura, transporte, reciclagem e descarte. Em relação aos dados de entrada referentes ao sistema de aquecimento de água com utilização do queimador atmosférico para o processo de pasteurização, os processos foram selecionados da base de dados Ecoinvent (2014) e estão dispostos na Tabela 1.

Tabela 1. Processos selecionados para o sistema de referência (Boiler GLP)

\begin{tabular}{lc}
\hline \multicolumn{1}{c}{ Processo } & Quantidade \\
\hline Chapa de inox com revestimento de estanho & $2 \mathrm{~m}^{2}=5 \mathrm{~kg}$ \\
Fibra de vidro & $10 \mathrm{~kg}$ \\
Aço inox laminado a quente & $160 \mathrm{~kg}$ \\
Calor oriundo do GLP & $71.522,68 \mathrm{MJ} / \mathrm{kg}$ \\
\hline
\end{tabular}

${ }^{1}$ Neste processo, o propano é extraído do GLP, sendo composto em média de 60\%-85\% de propano, $14 \%-38 \%$ de butano e $0 \%-6 \%$ de outros gases. Uma eficiência média de $75 \%$ foi considerada para este processo (eficiência da caldeira).

Considerou-se o aproveitamento de $100 \%$ dos resíduos de aço e ferro para reciclagem, e que os resíduos remanescentes seriam descartados em aterro (já que o ferro e aço foram reciclados). Para o inventário da ACV do queimador atmosférico a GLP, foi considerada uma vida útil de 10 anos. Os dados de entrada referentes ao SAS também foram obtidos da base de dados Ecoinvent (2014), e estão mostrados na Tabela 2 .

Tabela 2. Processos selecionados para o SAS

\begin{tabular}{ll}
\hline Processo & Quantidade \\
\hline Tanque de água quente $(600 \mathrm{~L})$ & $260 \mathrm{~kg}$ \\
Coletor solar plano $^{2}$ & $12 \mathrm{~m}^{2}=96 \mathrm{~kg}$ \\
\hline
\end{tabular}

${ }^{2}$ Admite-se neste processo que o revestimento de cobre preto é de fabricação norte-americana. 
Neste processo foi considerado o aproveitamento de $100 \%$ dos resíduos de aço e ferro para reciclagem, como considerado para o sistema de referência. Definiu-se que outros resíduos seriam descartados em aterro. A vida útil deste sistema também foi de 10 anos.

\subsection{AVALIAÇÃO DOS IMPACTOS}

Essa fase tem a finalidade de compreender e analisar o impacto ambiental causado pelo consumo de matéria-prima e a emissão dos rejeitos identificados no momento do inventário. O mais importante nessa fase é a redução da poluição originada por um determinado processo em que um dos fatores responsáveis para tal é a utilização indiscriminada de elementos ecológicos, implicando em um alto grau de degradação ambiental (GIANNETTI; ALMEIDA, 2006).

Para o processamento dos dados coletados em análise, foi utilizado o software SimaPro ${ }^{\circledR}$ versão 8.0.3.14 (PRÉCONSULTANTS, 2014a). O SimaPro ${ }^{\circledR}$ oferece diversos métodos para o cálculo de impactos ambientais, dentre os quais foi selecionado o método IPCC 2013 GWP 100a, um dos mais utilizados para análise de impactos ambientais, expressando o impacto em termos de quilogramas de dióxido de carbono-equivalente ( $\mathrm{kg} \mathrm{CO}_{2}$-eq). Este método utiliza os fatores de conversão atualizados do IPCC para a quantificação das contribuições de emissões atmosféricas para o problema das mudanças climáticas, e caracteriza as diferentes emissões gasosas de acordo com o seu potencial de aquecimento global em 100 anos (IPCC, 2013), proporcionando a pegada de carbono.

A pegada de carbono de produtos pode ser muito útil para as organizações, já que a partir de seu cálculo podem-se estabelecer metas e programas de redução e demonstrar aos clientes a inserção da variável ambiental no negócio. O consumidor está cada vez mais consciente de seus impactos ambientais e hoje em dia possui mais acesso à informação e mais poder de pressão sobre as marcas, exigindo práticas e valores alinhados aos seus (CARVALHO; FREIRE; MAGNO, 2015). 


\section{6 ANÁLISE DE SENSIBILIDADE}

Foi realizada a análise de sensibilidade para examinar diferentes cenários em relação às pegadas de carbono. Para Blank e Tarquin (2008), tais análises permitem verificar os efeitos finais considerando uma determinada variável, dada uma modificação em suas quantidades, assim gerando projeções futuras para o processo de tomada de decisões.

Para este trabalho, foi utilizada uma variação de $\pm 2 \mathrm{~m}^{2}$ no tamanho do SAS. As simulações foram realizadas de forma individualizada, estudando o efeito isolado de cada uma delas, ou seja, era alterada apenas uma variável e coeteris paribus para as demais.

\section{RESULTADOS E DISCUSSÃO}

A Tabela 3 refere-se aos dados do consumo de GLP pelo queimador atmosférico no processo de pasteurização para a produção de picolé e sorvete na empresa no período que abrangeu fevereiro de 2013 a maio de 2015.

Tabela 3. Consumo Mensal de GLP entre 2013 e 2015

\begin{tabular}{l|ccc}
\hline \multirow{2}{*}{ Meses } & \multicolumn{3}{|c}{ Consumo de GLP (kg) } \\
& $\mathbf{2 0 1 3}$ & $\mathbf{2 0 1 4}$ & $\mathbf{2 0 1 5}$ \\
\hline Janeiro & - & 356 & - \\
Fevereiro & 230 & 122 & 173 \\
Março & - & 94 & 258 \\
Abril & 195 & 177 & - \\
Maio & - & - & 168 \\
Junho & 124 & 100 & - \\
Julho & 176 & 136 & - \\
Agosto & 140 & 177 & - \\
Setembro & 193 & 87 & - \\
Outubro & 191 & - & - \\
Novembro & 306 & - & - \\
Dezembro & 104 & 94 & $\mathbf{5 9 9}$ \\
\hline Total & $\mathbf{1 6 5 9}$ & $\mathbf{1 3 4 3}$ & \\
\hline
\end{tabular}


Foi observado que o maior consumo anual de GLP foi no ano de 2013. No mês de janeiro de 2014 foi apresentado o maior consumo da série, com $356 \mathrm{~kg}$. Em 2013 e 2014, em média, foram consumidos anualmente $1.501 \mathrm{~kg}$ de GLP em 20 horas médias mensais de utilização do processo de pasteurização, levando em conta um consumo médio mensal de $171 \mathrm{~kg}$ para 9 meses do ano. Foi averiguado que a empresa não apresenta consumo constante de GLP entre os meses, demonstrando a sazonalidade na produção de sorvetes e picolés.

A Figura 2 apresenta a relação entre as vendas de picolés e sorvetes no período de 2013 a 2015 e o consumo de GLP no mesmo período. O eixo vertical esquerdo caracteriza as unidades de vendas dos produtos, já o eixo vertical direito expressa o consumo anual de GLP em kg. Observa-se uma relação positiva entre volume demandado pelos pedidos e o consumo do GLP, uma vez que apenas esses dois produtos são submetidos ao processo de pasteurização utilizando do queimador atmosférico.

A Figura 3 apresenta as pegadas de carbono anuais associadas ao processo de pasteurização com queimador atmosférico e com SAS, totalizando, respectivamente, $2090 \mathrm{~kg} \mathrm{CO}$-eq/ano e $142 \mathrm{~kg} \mathrm{CO}_{2}$-eq/ano. As pegadas de carbono possuem duas componentes: uma material, relacionada à construção/fabricação do equipamento (e que é dividida pela vida útil de cada sistema), e uma operacional, que compreende os fluxos energéticos necessários para seu funcionamento. Na Figura 3 apresentamse diagramas de Sankey, na qual a espessura das linhas conectoras representa a magnitude do impacto ambiental. A reciclagem é traduzida como impactos evitados (por evitar a produção de determinado material) e por isso as conexões são verdes. 


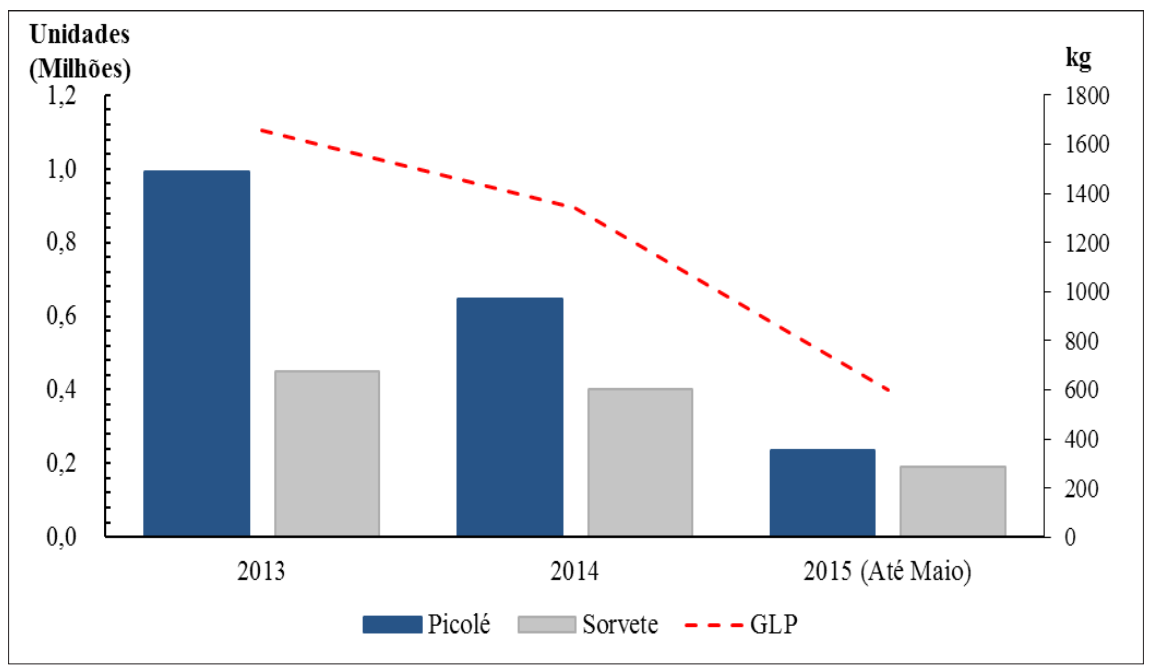

entre 2013 e 2015.

É possível observar na Figura 3 que a maior parte da pegada de carbono associada ao uso do queimador atmosférico é devida à queima do GLP (171 kg de GLP em 9 meses), sendo produzidos $2.100 \mathrm{~kg} \mathrm{CO}_{2}$-eq/ano. Entretanto, com a reciclagem dos resíduos de ferro e aço fundidos, ocorre uma redução de $36,7 \mathrm{~kg}$ $\mathrm{CO}_{2}$-eq por ano. No total, são produzidos $2.090 \mathrm{~kg} \mathrm{CO}$-eq/ano

Ao ser utilizado um SAS, fica explicitado pela Figura 3 b que a maior pegada de carbono está relacionada às placas coletoras, apresentando uma geração anual de $142,1 \mathrm{~kg}$ de $\mathrm{CO}_{2}$-eq, enquanto o reservatório de água quente exibiu um impacto anual de $74,8 \mathrm{~kg}$ de $\mathrm{CO}_{2}$-eq. Somados, apresentam um impacto equivalente a $217 \mathrm{~kg}$ de $\mathrm{CO}_{2}$-eq. No entanto, em decorrência da reciclagem do ferro e do aço, são poupados $74,7 \mathrm{~kg}$ de $\mathrm{CO}_{2}$-eq por ano, totalizando $142 \mathrm{~kg} \mathrm{CO}_{2}$-eq dos impactos ambientais associados ao uso do queimador de GLP e SAS e resultando em uma redução anual de 93\% da emissão de $\mathrm{CO}_{2}$-eq somente neste processo de pasteurização. 


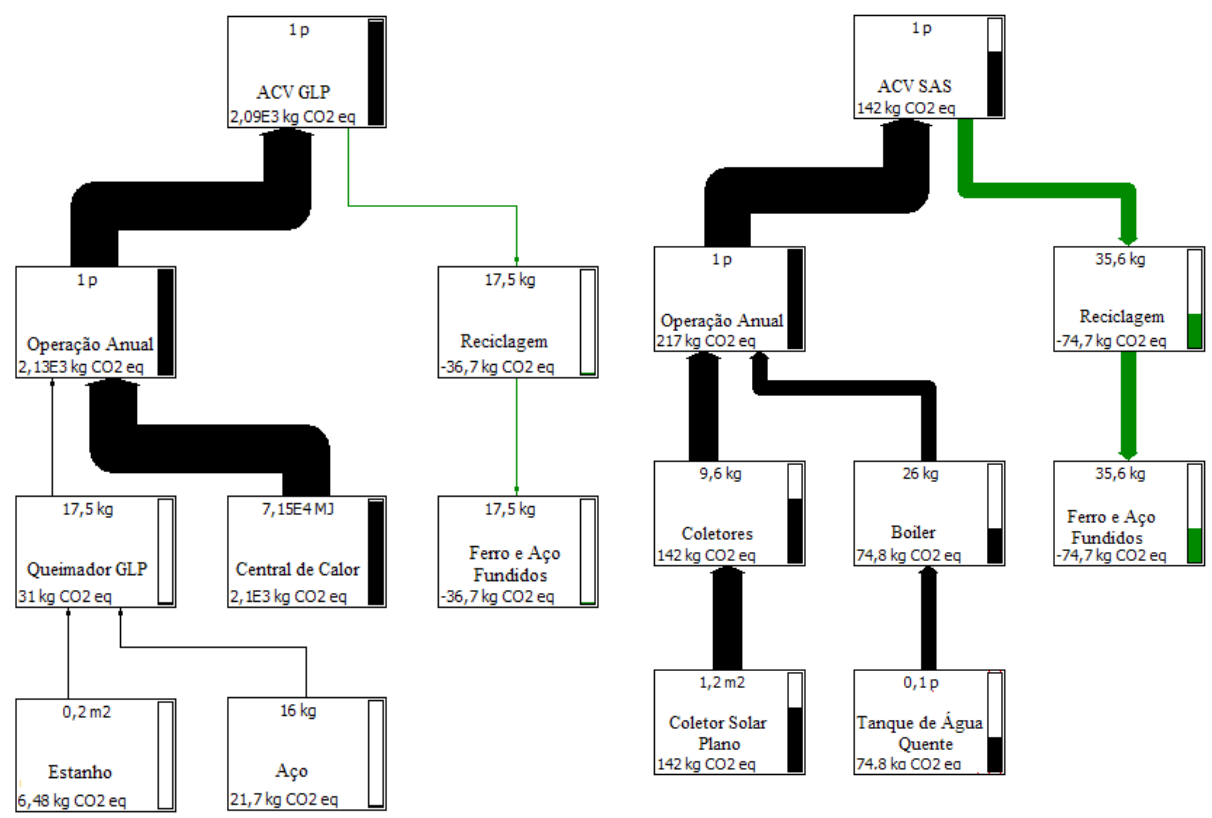

Figura 3. Impactos Ambientais Anuais Associados ao Processo de Pasteurização com Queimador Atmosférico (a) e SAS (b), com Método IPCC 2013 GWP 100a.

\subsection{ANÁLISE DE SENSIBILIDADE DE CENÁRIOS}

A Tabela 4 apresenta a análise de sensibilidade nas pegadas de carbono, para os valores anuais de consumo de GLP pelo queimador atmosférico. Foi atestado que a cada $\pm 10 \%$ de impacto no consumo do GLP, serão afetados três vezes os níveis de pegada de carbono, ou seja, aproximadamente de $\pm 210 \mathrm{~kg} \mathrm{CO}_{2}$-eq.

A Tabela 5 apresenta a análise de sensibilidade considerando valores anuais para o SAS com maior área útil de operação. A cada ampliação ou redução da área coletora em 10\%, os impactos sofrerão um aumento de cerca de $10 \mathrm{~kg} \mathrm{CO}_{2}$-eq por ano. A pegada de carbono conseguiu evidenciar que do ponto de emissões de GEE, a utilização de um SAS (energia solar) é melhor do que a utilização de GLP em um queimador durante o processo de pasteurização em uma indústria de sorvetes localizada na Paraíba. 
Tabela 4. Pegada de carbono anual para uma variação do consumo do GLP no queimador atmosférico

\begin{tabular}{cc}
\hline Variação (\%) & Pegada de carbono $\left(\mathrm{kg} \mathrm{CO}_{2}\right.$-eq/ano) \\
\hline-30 & 1460 \\
-20 & 1670 \\
-10 & 1880 \\
0 & 2090 \\
10 & 2300 \\
20 & 2510 \\
30 & 2720 \\
\hline
\end{tabular}

Tabela 5. Pegada de carbono anual para uma ampliação da área útil de utilização do SAS

\begin{tabular}{|c|c|}
\hline Variação (\%) & Pegada de carbono ( $\mathrm{kg} \mathrm{CO}_{2}$-eq/ano) \\
\hline-20 & 122 \\
\hline-10 & 132 \\
\hline 0 & 142 \\
\hline 10 & 152 \\
\hline 20 & 162 \\
\hline
\end{tabular}

A substituição da queima do GLP por um SAS possui potencial de mitigação de mudanças climáticas, em virtude da diferença de pegadas de carbono obtidas. Por ano, com a implementação do SAS, obtêm-se emissões evitadas da ordem de 2090 - $142=-1948 \mathrm{~kg} \mathrm{CO}$-eq/ano. Este valor é aplicável para uma microempresa, e pode ser extrapolado para indústrias maiores no Brasil, considerando-se uma composição fixa para o GLP. As emissões do SAS estão associadas à sua construção, não havendo pegada de carbono associada à sua operação e manutenção. Por outro lado, o sistema de referência apresenta considerável pegada de carbono associada à sua operação (queima de GLP). Ambas pegadas de carbono associadas à construção do sistema (composição material) são amortizáveis ao longo de sua vida útil, e por isso resultam em valores baixos neste caso.

A ACV vem sendo, pouco a pouco, mais utilizada em diferentes indústrias alimentícias para quantificação de impactos ambientais. Na literatura internacional, Roy et al. (2009) realizaram uma revisão sobre a ACV de alguns produtos alimentícios, 
que incluiu alguns alimentos industrializados, a produção de laticínios e carne, e de outros produtos agrícolas. Trabalhos mais recentes são sobre o pão (ESPINOZAORIAS; STICHNOTHE; AZAPAGIC, 2011), sorvete (BEN; JERRY'S, 2016), chocolate (ESU-SERVICES, 2016), e doces, salgadinhos e refrigerantes (NILSSON; SUND; FLÓREN, 2011).

Especificamente no Brasil, Melquíades et al. (2015) estudaram o processo produtivo do pão francês em uma padaria paraibana, concluindo que o gás natural participou do mix energético da panificadora em maior porcentagem e custos e, em contrapartida, o consumo do briquete de bagaço de cana demonstrou baixos níveis de impactos ambientais, além do preço reduzido. O trabalho de Silva et al. (2016) quantificou a pegada de carbono associada a dois sabores de bolos produzidos por uma pequena fábrica paraibana, já pensando na possível aplicação de etiquetas ecológicas que informem ao consumidor as emissões embutidas em um determinado produto. A ACV foi aplicada também em outros trabalhos brasileiros publicados no setor alimentício, ou ao processo produtivo como um todo ou a produtos específicos. Carvalho, Grilo e Abrahão (2017) verificaram duas formas de produção caseira para chips de batata, concluindo que a utilização de fritura convencional em fogão com óleo de soja possuía maior pegada de carbono que a utilização de equipamento do tipo air-fryer.

O trabalho de Willers et al. (2017) desenvolveu uma ACV para a produção de carne bovina no Nordeste brasileiro, enquanto a pecuária bubalina foi o tema de Soares et al. (2016). Santos et al. (2017) desenvolveram uma ACV para o processo produtivo do queijo em uma empresa de pequeno porte na Bahia, e Ferraz et al. (2017) avaliaram os impactos ambientais associados à produção de ovos na Bahia. A avaliação e proposta de mitigação dos impactos ambientais potenciais associados à produção de queijo prato na Bahia foi proposta por Ferreira, Rodrigues e Robra (2017), enquanto o iogurte foi estudado por Ferreira et al. (2016) e Santos Junior et al. (2016a), o queijo por Santos Junior et al. (2016b), o leite por Carvalho et al. (2016).

Compreender os efeitos ambientais associados a diferentes alternativas para processos é crítico para caracterizar a sustentabilidade, ajudar a elaborar políticas públicas e normativas, e informar o consumidor. 


\section{CONCLUSÕES}

A partir das análises realizadas foi possível concluir que:

O Sistema de Aquecimento Solar (SAS) proporcionou menor pegada de carbono, quando comparado ao queimador atmosférico (que utiliza GLP).

A pegada de carbono associada ao uso de queimador atmosférico foi em sua maioria associada ao consumo de GLP no queimador, totalizando $2.090 \mathrm{~kg} \mathrm{CO}_{2}$ eq/ano, com $-36,7 \mathrm{~kg} \mathrm{CO}_{2}$-eq/ano devido ao processo de reciclagem do aço e ferro fundido.

A pegada de carbono associada ao Sistema de Aquecimento Solar foi de 142 $\mathrm{kg} \mathrm{CO}_{2}$-eq/ano para um sistema de $12 \mathrm{~m}^{2}$ de área útil e boiler de 600 litros.

Mesmo com a elevação da área coletora do SAS, a pegada de carbono resultante foi inferior ao aumento ou redução no consumo do GLP, já que seu efeito seria ampliado três vezes para $\pm 10 \%$ de seu uso.

\section{AGRADECIMENTOS}

Os autores agradecem o apoio do Conselho Nacional de Desenvolvimento Científico e Tecnológico (Projetos $n^{0} 406788 / 2013-8$, $n^{\circ} 475879 / 2013-9$ e $n^{0}$ 303199/2015-6).

\section{REFERÊNCIAS}

ABNT. Associação Brasileira de Normas Técnicas. NBR. 15569/2008: Sistema de aquecimento solar de água em circuito direto. Rio de Janeiro: ABNT, 2008.

ABNT. NBR ISO 14040/2014 - Gestão ambiental - avaliação do ciclo de vida: princípios e estrutura. Rio de Janeiro: ABNT, $2014 \mathrm{a}$.

ABNT. NBR ISO 14044/2014 - Avaliação do ciclo de vida: requisitos e orientações. Rio de Janeiro: ABNT, $2014 \mathrm{~b}$. 
ABRAVA - Associação Brasileira de Refrigeração, Ar Condicionado, Ventilação e Aquecimento. Produção de coletores solares para aquecimento de água e reservatórios térmicos no Brasil: ano de 2014. São Paulo, 2015. Disponível em: http://www.solarthermalworld.org/sites/gstec/files/news/file/2015-07-27/market statistics_2014_brazil.pdf. Acesso em: 17 jul. 2017.

ARAÚJO, Y. R. V.; GÓIS, M. L.; COELHO JUNIOR, L. M.; CARVALHO, M. Carbon footprint associated with four disposal scenarios for urban pruning waste.

Environmental Science and Pollution Research, v. 25, n. 2, p. 1863-1868, 2018.

BEN; JERRY'S. A life cycle analysis study of some of our flavors (2016). Disponível em: http://www.benjerry.com/values/issues-we-care-about/climate-justice/life-cycleanalysis. Acesso em: 12 mar. 2018.

BLANK, L.; TARQUIN, A. Engenharia econômica. 6a ed. São Paulo: McGraw-Hill, 2008.

BRASIL. Agência Nacional de Vigilância Sanitária- ANVISA. Resolução RDC no 267. Regulamento técnico de boas práticas de fabricação para estabelecimentos industrializadores de gelados comestíveis. Brasília, 2003.

BRASIL. Ministério do Trabalho e Emprego - MTE. Relação Anual de Informações Sociais - RAIS. Disponível em: http://portal.mte.gov.br/rais/. Acesso em: 17 jul. 2017.

CARVALHO, L. S.; WILLERS, C. D.; ROBRA, S.; ALMEIDA NETO, J. A.; RODRIGUES, L. B. Emissões de Gases de Efeito Estufa da produção de leite em uma fazenda do Território de Identidade Médio Sudoeste da Bahia. In: CBGCV - CONGRESSO BRASILEIRO EM GESTÃO DO CICLO DE VIDA, 5., 2016, Fortaleza. Anais [...]. Fortaleza: IBICT, 2016.

CARVALHO, M.; ABRAHAO, R. Environmental and economic perspectives in the analysis of two options for hand drying. International Journal of Emerging Research in Management and Technology, v. 7, p. 24-35, 2017.

CARVALHO, M.; FREIRE, R. S.; MAGNO, A. H. Promotion of sustainability by 
quantifying and reducing the carbon footprint: new practices for organizations. In: GLOBAL CONFERENCE ON GLOBAL WARMING, 2., 2015, Atenas. Anais [...]. Atenas: CERTH: 2015.

CARVALHO, M.; GRILO, M. M. S.; ABRAHAO, R. Comparison of greenhouse gas emissions relative to two frying processes for homemade potato chips.

Environmental Progress \& Sustainable Energy, v. 37, n. 1, p. 481-487, 2018.

DINATO, R. M. Sistematização dos métodos de contabilização de emissões de gases de efeito estufa sob a ótica do ciclo de vida. 2013. 86f. Dissertação (Mestrado em Engenharia Química) - Universidade de São Paulo, São Paulo. 2013.

ECOINVENT. The ecoinvent data base. 2015. Disponível em: http://www. ecoinvent.org/. Acesso em: 17 jul. 2017.

EPE - Empresa de Pesquisa Energética. Balanço energético nacional 2015: ano base 2014. Rio de Janeiro, 2015. Disponível em: https://ben.epe.gov.br/downloads/ Relatorio_Final_BEN_2015.pdf. Acesso em: 17 jul. 2017.

ESPINOZA-ORIAS, N.; STICHNOTHE, A. A. The carbon footprint of bread. The International Journal of Life Cycle Assessment, v. 16, n. 4, p. 351-365, 2011.

ESU- SERVICES. Life cycle assessment of Swiss chocolate, 2016. Disponível em: http://www.esu-services.ch/fileadmin/download/jungbluth-2014-SETAC-chocolate. pdf. Acesso em: 12 mar. 2018.

FERRAZ, S. P.; WILLERS, C. D.; ALVARENGA, R. A. F.; ALMEIDA NETO, J. A.; RODRIGUES, L. B. Environmental Impacts Assessment of Eggs Production in Bahia, Brazil. In: CILCA - CONFERENCIA INTERNACIONAL DE ANÁLISIS DE CICLO DE VIDA EN LATINOAMÉRICA, 7., 2017, Medelin. Proceedings [...]. 2017.

FERREIRA, F. U.; ROBRA, S.; ALMEIDA NETO, J. A.; RODRIGUES, L. B. Identificação e proposta de mitigação dos impactos ambientais da produção de iogurte. In: CBGCV - CONGRESSO BRASILEIRO EM GESTÃO DO CICLO DE VIDA, 5., 2016, Fortaleza. Anais [...]. Fortaleza: IBICT, 2016. 
FERREIRA, F. U.; RODRIGUES, L. B.; ROBRA, S. Avaliação e proposta de mitigação dos impactos ambientais potenciais associados à produção de queijo prato. In: ENEGEP - ENCONTRO NACIONAL DE ENGENHARIA DE PRODUÇÃO, 36., 2016, João Pessoa. Anais [...]. João Pessoa: ABEPRO, 2016.

FREIRE, R. S.; CARVALHO, M.; CARMONA, C. U. M.; MAGNO, A. H. Perspectives on the implementation of climate change public policies in Brazil. In: GLOBAL CONFERENCE ON GLOBAL WARMING, 2., 2015, Atenas. Anais [...]. CERTH: Atenas, 2015.

GIANNETTI, B. F.; ALMEIDA, C. M. B. V. Ecologia Industrial: conceitos, ferramentas e aplicações. São Paulo: Edgard Blucher, 2006.

GRILO, M. M. S.; FORTES, A. F. C.; SOUZA, R. P. G.; SILVA, J. A. M.; CARVALHO, M. Carbon footprints for the supply of electricity to a heat pump: Solar energy vs. electric grid. Journal of Renewable and Sustainable Energy 10, $023701,2018$. DOI: https://doi.org/10.1063/1.4997306.

GUINÉE, J. B. (Ed.). Life Cycle Assessment: An operational guide to the ISO Standards; LCA in Perspective; Guide; Operational Annex to Guide. Centre for Environmental Science, Leiden University, The Netherlands, 2001.

GUINÉE, J. B. Handbook on life cycle assessment: operational guide to the ISO standards. Boston: Kluwer Academic Publishers,2002.

IEA - International Energy Agency. World energy outlook 2013. Paris, 2013. Disponível em: http://www.worldenergyoutlook.org/media/weowebsite/2013/ WEO2013_Ch06_Renewables.pdf Acesso em: 17 jul. 2017.

IEA. $\mathrm{CO}_{2}$ emissions from fuel combustion. Paris, 2014a. Disponível em: https://www.iea.org/publications/freepublications/publication/ CO2EmissionsFromFuelCombustionHighlights2014.pdf Acesso em: 17 jul. 2017.

IEA. Key world energ y statistics. Paris, 2014b. Disponível em: http://www.iea. org/publications/freepublications/publication/keyworld2014.pdf Acesso em: 17 jul. 2017. 
INTERNATIONAL ORGANIZATION FOR STANDARDIZATION (ISO), Genebra, 2006.

IPCC - INTERGOVERNMENTAL PANEL ON CLIMATE CHANGE. Revised supplementary methods and good practice guidance arising from the Kyoto protocol. 2013. Disponível em: http://www.ipcc-nggip.iges.or.jp/public/kpsg/. Acesso em: 01 mar. 2016.

IPCC - Intergovernmental Panel on Climate Change. Anthropogenic and Natural Radiative Forcing. 2014. Disponível em: https://www.ipcc.ch/pdf/assessmentreport/ar5/wg1/WG1AR5_Chapter08_FINAL.pdf. Acesso em: 17 jul. 2017.

ISO 14040. Environmental management - Life cycle assessment - Principles and framework. ISO 14044. Environmental management - Life cycle assessment - Requirements and guidelines. International Organization for Standardization (ISO), Genebra, 2006.

MACHADO, M. G. C. Modelização do ciclo de vida do vinho verde e da rolha de cortiça natural por utilização do SimaPro recorrendo a vários métodos de avaliação de impactos ambientais. 2008. 88f. Dissertação (Mestrado em Engenharia Mecânica de Materiais) - Universidade Tecnológica Federal do Paraná, Curitiba. 2008.

MELQUIADES, T. F.; COELHO, I. M. A.; COELHO JUNIOR, L. M.; CARVALHO, M. Quantificação dos impactos ambientais do processo produtivo do pão francês. In: CONGRESSO INTERNACIONAL DE BIOENERGIA, 10., 2015, São Paulo. Anais [...]. São Paulo: [s. n.], 2015.

MENEZES, H. A. F.; CARVALHO, M.; FREIRE, R. S. Identificação de boas práticas para implementação da análise do ciclo de vida na Paraíba. In: CONGRESSO INTERNACIONAL DE BIOENERGIA, 10., 2015, São Paulo. Anais [...]. São Paulo: [s. n.], 2015.

NEVES, T. I.; UYEDA, C. A.; CARVALHO, M.; ABRAHÃO, R. Environmental evaluation of the life cycle of elephant grass fertilization - Cenchrus purpureus (Schumach.) Morrone - using chemical fertilization and biosolids. Environmental monitoring and assessment, v. 190, n. 1, p. 30, 2018. 
NILSSON, K.; SUND, V.; FLORÉN, B. The environmental impact of consumption of sweets, crisps and soft drinks. Copenhagen: Tema Nord, 2011.

PRÉCONSULTANTS. SimaPro Database Manual Methods library. RéConsultants, 2014b.

PRÉCONSULTANTS. Software SimaPro. 2014a. Disponível em: http://www.pre.nl/ simapro. Acesso em: 17 jul. 2017.

ROY, P.; NEI, D.; ORIKASA, T.; XU, Q.; OKADOME, H.; NAKAMURA, N.; SHIINA, T. A review of life cycle assessment (LCA) on some food products. Journal of food engineering, v. 90, n. 1, p. 1-10, 2009.

SANTOS JUNIOR, H. C. M.; MARANDUBA, H. L.; ALMEIDA NETO, J. A.; RODRIGUES, L. B. Life cycle assessment of cheese production process in a small-sized dairy industry in Brazil. Environmental Science and Pollution Research, v. 24, n. 4, p. 3470-3482, 2017.

SANTOS JUNIOR, H. C. M.; SANTOS, B. A.; MARANDUBA, H. L.; ALMEIDA NETO, J. A.; RODRIGUES, L. B. Impactos Ambientais no Ciclo de Vida de Produtos Lácteos: O Caso do Iogurte. In: CBGCV - CONGRESSO BRASILEIRO EM GESTÃO DO CICLO DE VIDA, 5., 2016, Fortaleza. Anais [...]. Fortaleza: IBICT, 2016.

SANTOS JUNIOR, H. C. M.; SANTOS, B. A.; MARANDUBA, H. L.; ALMEIDA NETO, J. A.; RODRIGUES, L. B. Impactos Ambientais no Ciclo de Vida de Produtos Lácteos: O Caso do Queijo. In: CBGCV - CONGRESSO BRASILEIRO EM GESTÃO DO CICLO DE VIDA, 5., 2016, Fortaleza. Anais [...]. Fortaleza: IBICT, 2016.

SCHNITZER, H.; BRUNNER, C.; GWEHENBERGER, G. Minimizing greenhouse gas emissions through the application of solar thermal energy in industrial processes. Journal of Cleaner Production, v. 15, n. 13, p. 1271-1286, 2007.

SEBRAE - Serviço Brasileiro de Apoio às Micro e Pequenas Empresas. Ideias de negócios para 2014: fabricação de sorvetes e outros gelados comestíveis. Brasília, 2012. Disponível em: http://segmentos.sebrae2014.com.br/ideiasdenegocios/ fabricacao-de-sorvetes-e-outros-gelados-comestiveis/ $: \mathrm{id}=8784 \& \mathrm{t}=-1$. Acesso em: 20 jul. 2015. 